\title{
GEOGEBRA 3D: UMA FERRAMENTA PARA ESTUDO DE VOLUMES NO ENSINO MÉDIO
}

\author{
José Carlos de SOUZA JÚNIOR ${ }^{1}$ \\ Andréa CARDOSO ${ }^{2}$ \\ Rejiane Aparecida CALIXTO³
}

1 - Licenciado e bacharel em Matemática pelo ICMC-USP, mestre e doutor em Matemática pela mesma instituição Atualmente é professor do curso de Licenciatura em Matemática do Instituto de Ciências Exatas da Universidade Federal de Alfenas (UNIFAL-MG). Contato: jose.souza@unifal-mg.edu.br

2 - Licenciada em Matemática pela UNESP, mestre em Matemática pelo ICMC-USP e doutora em Engenharia Mecânica pela EESC-USP. Atualmente é professora do curso de Licenciatura em Matemática do Instituto de Ciências Exatas da Universidade Federal de Alfenas (UNIFAL-MG). Contato: andreac74@uol.com.br

3 - Licenciada em Matemática pela Universidade Federal de Alfenas (UNIFAL-MG). Foi bolsista de iniciação à docência da área de Matemática do PIBID/UNIFAL-MG atuando em uma escola pública do estado de Minas Gerais no ensino médio. Contato: rejicalixto@hotmail.com

Recebido em: 23/05/2014 - Aprovado em: 30/06/2014 - Disponibilizado em: 30/07/2014

Resumo: Este artigo apresenta objetos de aprendizagem, construídos com o auxílio do software de geometria dinâmica GeoGebra juntamente com os resultados da aplicação de uma sequência didática utilizando esses objetos. Tendo como objetivo a motivação e complementação do estudo do volume dos principais sólidos geométricos abordados nos livros didáticos do ensino médio. A construção dos objetos aqui apresentados está respaldada na metodologia da visualização e experimentação, onde o aluno é incentivado e tem condições de tirar suas próprias conclusões, de modo a contribuir para o processo de ensino-aprendizagem. $\mathrm{O}$ uso desses objetos contribuiu para o entendimento dos tópicos estudados, a partir da visualização dos objetos geométricos.

Palavras-chave: Ensino de Matemática, Objetos de Aprendizagem, Geometria Espacial.

\section{GEOGEBRA 3D: A TOOL FOR THE STUDY OF VOLUMES IN HIGH SCHOOL}

\begin{abstract}
This paper presents learning objects, built with the aid of dynamic geometry software GeoGebra along with the results of the application of an instructional sequence using these objects. Aiming the motivation and complementation of a study of the volume of the main geometric solids addressed in high school textbooks. The construction of the objects presented here is based in methodology of visualization and experimentation, where the student is encouraged and is able to draw their own conclusions, so as to contribute to the process of teaching-learning. The use of these objects contributed to understanding of the topics studied, from the visualization of geometric objects. Keywords: Mathematics Teaching, Learning Objects, Spatial Geometry.
\end{abstract}




\section{1 - Introdução}

É impossível ignorar as novas tecnologias da informação e da comunicação (TIC) que transformaram a sociedade e ainda estão a modificar os meios de comunicação, o ambiente de trabalho e o próprio pensamento humano. Nas últimas décadas, o recurso computacional passou a receber maior destaque na educação, não somente pela demanda da sociedade moderna, altamente tecnológica, mas também devido a seu potencial pedagógico.

Em particular, determinados conceitos matemáticos podem ser compreendidos pela visualização e experimentação com o apoio de programas computacionais específicos. A representação de objetos tridimensionais no plano é uma das principais dificuldades dos professores de Matemática quando propõem o estudo de Geometria Espacial. O objeto representado no papel nem sempre corresponde à formação da imagem mental que se tem dele, dificultando assim a visualização e a compreensão por parte dos alunos.

Esta pesquisa busca responder quais são as contribuições dos recursos computacionais para a aprendizagem de Geometria Espacial Métrica. Assim, o objetivo deste trabalho é avaliar a aprendizagem dos alunos em intervenções pedagógicas pautadas pela aprendizagem significativa (Ausubel, 1980), utilizando material didático digital especialmente desenvolvido para o estudo de volume dos principais sólidos geométricos e dedução das fórmulas comumente utilizadas no ensino básico.

\section{2 - Computadores e Geometria}

Os programas computacionais apresentam potencialidades para o ensino de matemática, principalmente para a aprendizagem de geometria. Especificamente, os recursos computacionais destinados ao ensino de geometria espacial permitem à estudantes e professores a construção rápida e precisa de figuras geométricas, permitindo ainda a exploração de formas, propriedades e invariâncias dos objetos.

Engers et al. (2004) apresenta uma proposta pedagógica para o ensinoaprendizagem da geometria nas séries iniciais utilizando o software LOGO3D, conclui que o computador pode ser um forte e versátil instrumento na área da educação, desde que utilizado de forma inteligente e competente.

Dentre os aplicativos para a aprendizagem de geometria, têm-se os Programas de Geometria Dinâmica (PGD), essencialmente baseados na geometria euclidiana. Este tipo de aplicativo é definido por um conjunto de objetos (pontos, retas, segmentos e circunferências) e ações elementares de construir, desenhar, medir, girar, refletir, transladar e movimentar, que 
permitem fazer construções geométricas rápidas e precisas. A característica fundamental dos programas de geometria dinâmica é a atualização automática de valores, como ângulos, distâncias e áreas, quando a figura é movimentada. Os PGD permitem a deformação das figuras mantendo as relações entre os objetos, e quando a figura geométrica é construída com base em suas propriedades, a movimentação da figura possibilita a percepção de padrões e invariâncias. Assim o aprendiz é capaz de fazer conjecturas e testá-las no próprio programa.

A exploração dos objetos geométricos nos PGD estimula o raciocínio, favorecendo a descoberta de novas relações e conceitos geométricos. Alves, Soares e Lima (2005) demonstram que o uso de PGD melhorou significativamente o desempenho dos alunos.

Dentre os diversos PGD disponíveis, alguns permitem a construção e visualização de objetos tridimensionais (3D), é o caso do software proprietário Cabri-Géomètre 3D que, além de preservar as propriedades de objetos geométricos tridimensionais, permite mudar o ponto de vista em relação ao objeto representado. Jahn e Bongiovanni (2009) discutem a construção e manipulação de poliedros utilizando o Cabri 3D.

Alves (2007) apresenta uma sequência didática para justificar as fórmulas de volume dos principais sólidos geométricos a partir do princípio de Cavalieri utilizando o software livre Calques 3 $\mathrm{D}^{1}$, destinado à aprendizagem da geometria espacial. O Calques 3D é um micro mundo planejado para a construção, observação e exploração de figuras geométricas espaciais.

O PGD GeoGebra é uma ferramenta computacional livre com potencial didático para o estudo de diversos conceitos matemáticos. O GeoGebra pode ser utilizado em laboratórios de informática das escolas públicas, visto que há um versão online sem necessidade de instalação em máquina ou ainda pode ser executado a partir de uma entrada USB, garantindo importantes características de portabilidade. A versão GeoGebra 5.0 $0^{2}$ conhecido como GeoGebra 3D, permite também a construção, visualização e exploração de objetos tridimensionais.

O GeoGebra permite a criação de objetos de aprendizagem para experimentação e exploração de determinados conceitos matemáticos por meio de recursos digitais dinâmicos e interativos (CARDOSO e SOUZA JR, 2011), e que podem ser disponibilizados no formato de applets, entendidos como programas interativos que podem ser executados em qualquer browser, sem necessidade de instalação na máquina do usuário.

Tarouco e colaboradores (2011) definem Objeto de Aprendizagem (OA) como

\footnotetext{
${ }^{1}$ Disponível para download em: http://www.uff.br/calques3d/ 2 Disponível em: http://www.geogebra.org/webstart/5.0/geogebra-50.jnlp
} 
"qualquer recurso digital que possa ser usado e reutilizado em situações de apoio ao ensino e aprendizagem". Assim, aliado às potencialidades dos PGD, OAs podem ser desenvolvidos em PGD e disponibilizados para manipulação direta dos estudantes, sem necessidade de realizar as construções, uma vez que o objetivo é a experimentação de conceitos e não o domínio técnico do programa computacional.

Neste sentido, os PGD podem e devem ser utilizados na elaboração de OAs que complementem o material disponível no livro didático e seja de livre e fácil acesso para estudantes e professores.

\section{3 - Produção dos Objetos de Aprendizagem}

Para o desenvolvimento dos OA, foi feita inicialmente uma busca de programas computacionais de geometria dinâmica 3D, com potencial para o desenvolvimento das atividades. Explorando suas ferramentas, funcionalidades e limitações. Foram avaliados os aplicativos: Cabri 3D, Calques 3D e

GeoGebra 3D, optou-se por utilizar o Geogebra 3D.

A interface do Geogebra 3D apresenta duas janelas, a primeira parecida com as versões anteriores do GeoGebra em 2D, exibe o Campo de Entrada, Janela de Álgebra e Janela de visualização. A segunda janela apresenta o ambiente 3D. As construções realizadas no ambiente 3D são projetadas no plano $x y$ e aparece na janela de visualização 2D, como apresentado na Figura 1a.

O primeiro OA desenvolvido objetivou a apresentação da ideia intuitiva de volume, entendido como a quantidade de espaço ocupada por um sólido, mensurado por comparação com uma unidade de medida padronizada. Para tanto, o OA propõe atividades para obtenção do volume do cubo pelo total preenchimento de seu interior com as unidades de medida e seus submúltiplos. A Figura $1 b$ ilustra a obtenção do volume de um cubo de aresta 2,5 pelo preenchimento com

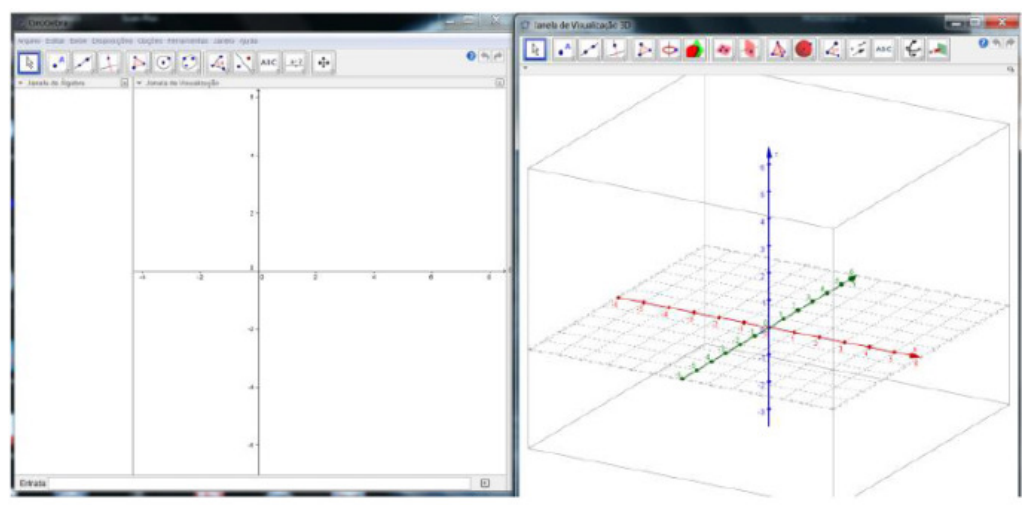

(a)

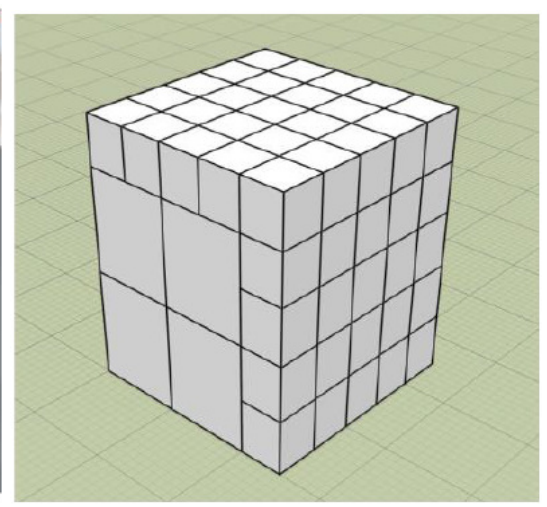

(b)

Figura 1: a) Janelas de visualização do GeoGebra 5.0; b) Visualização do volume do cubo de arestas 2,5 pelo preenchimento deste com cubos de aresta unitária e seus submúltiplos. 
cubos de aresta unitária e seus submúltiplos.

O cubo é um caso particular de bloco retangular com arestas de mesmo comprimento. Um bloco retangular é um sólido geométrico limitado por seis retângulos, dois a dois congruentes. Assim, a partir da ideia intuitiva de volume do cubo é possível generalizar e obter o volume do bloco retangular como área da base multiplicada pela altura.

A partir do volume do bloco

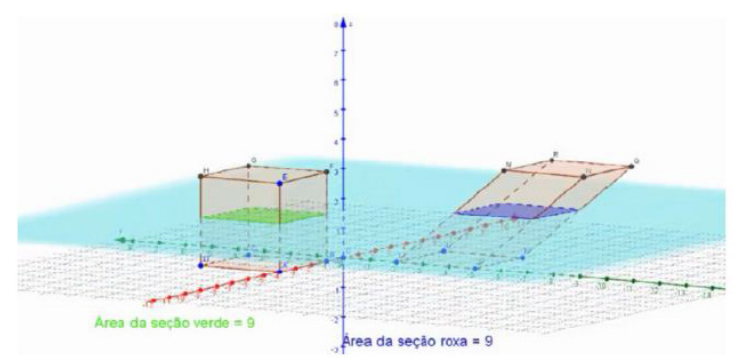

(a) demonstração, segundo Paterlini (2010) isso ocorre para que sejam evitadas as dificuldades de se apresentar precocemente a alunos do ensino médio, a teoria de integração de funções reais, necessária para a demonstração desse princípio.

Utilizando o princípio de Cavalieri conjuntamente com ferramentas adequadas ao estudo de volumes, é possível facilitar a visualização e levar os estudantes à compreensão das fórmulas de volume dos

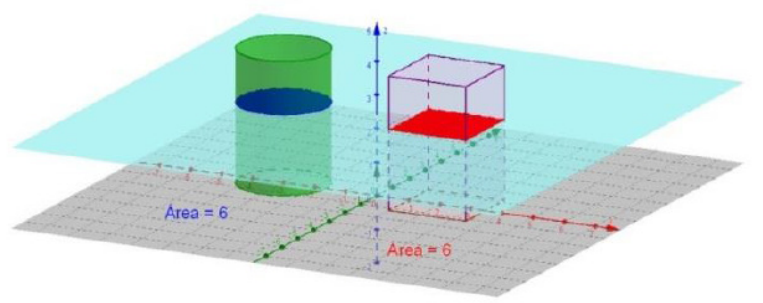

(b)

Figura 2: Aplicação do Princípio de Cavalieri na obtenção do volume (a) do paralelepípedo e (b) do cilindro.

retangular, as fórmulas de volume para paralelepípedos, prismas, pirâmides, cilindros e cones podem ser obtidas pelo Princípio de Cavalieri: se qualquer plano horizontal secciona dois sólidos A e B segundo figuras planas com áreas iguais, então o volume do sólido A é igual ao volume do sólido B.

Para Lima (2009), o Princípio de Cavalieri "permite uma simplificação notável nos argumentos que conduzem às fórmulas clássicas de volume", e é a base para o estudo de volumes de sólidos no ensino médio. Nos livros didáticos, esse resultado é adotado como postulado sem que se faça sua principais sólidos geométricos estudados na educação básica.

Para dedução do volume do paralelepípedo foi elaborado um OA apresentando um bloco retangular e um paralelepípedo cujas bases têm áreas iguais (Figura 2a). Por meio da ferramenta controle deslizante do GeoGebra é possível manipular o plano que intersecta esses sólidos, e o aluno pode constatar que as figuras planas formadas pela interseção do plano com os sólidos tem áreas equivalentes. A seguir, aplica-se o princípio de Cavalieri para deduzir que o volume do paralelepípedo também é obtido 

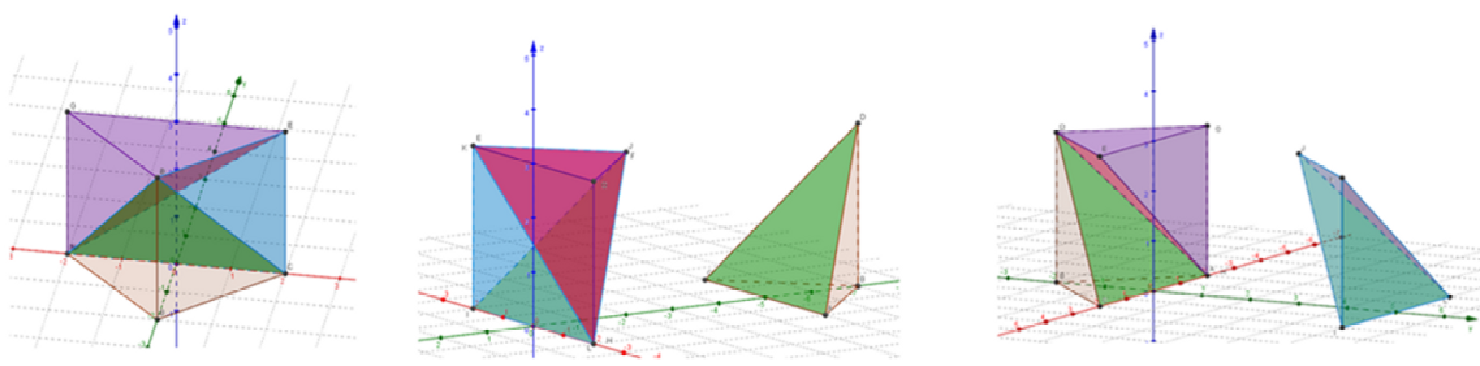

Figura 3: Prisma triangular reto decomposto em três pirâmides de volumes equivalentes.

pela multiplicação da área da base pela altura, visto que é sempre possível construir um bloco retangular com o mesmo volume que o paralelepípedo.

Para obter o volume do cilindro, a mesma ideia é utilizada, constroem-se um bloco retangular e um cilindro com áreas das bases iguais. Com a movimentação do plano paralelo ao plano da base pode-se observar que as áreas das figuras planas obtidas da interseção do plano com os sólidos em cada nível são iguais. Concluindo, pelo Princípio de Cavalieri, que o volume do cilindro é o mesmo do bloco retangular, ou seja, o produto da área da base pela altura. A Figura $2 \mathrm{~b}$ ilustra um instantâneo do OA para a o estudo do volume do cilindro. Também é possível deduzir o volume do prisma, de maneira mais geral.

Para o estudo do volume da pirâmide, foi elaborado um OA mostrando a decomposição de um prisma triangular em três pirâmides de mesmo volume, Figura 3. Controles deslizantes são utilizados para modificar a posição das pirâmides. Com valores iniciais $a=b=c=0$, pode-se observar o prisma triangular reto, enquanto que atribuindo os valores $a=2, \quad b=0$ e $c=2$ constata-se que a base IKL de uma das pirâmides é congruente à base EGH de outra pirâmide e que a altura da primeira, a partir do vértice $\mathrm{J}$, é igual à altura da segunda, a partir do vértice F. Logo, estas duas pirâmides têm o mesmo volume. Manipulando convenientemente os controles deslizantes é possível concluir que as três pirâmides possuem volumes iguais.

O volume do cone pode ser obtido também utilizando o Princípio de Cavalieri pela comparação com o volume da pirâmide. Um cone circular reto foi construído com a área da base e altura previamente fixadas, para em seguida construir uma pirâmide com uma das faces com área igual à base do cone $\mathrm{e}$ mesma altura do cone em relação a esta face. A movimentação do controle deslizante permite visualizar diversas seções planas com áreas equivalentes, resultantes da interseção do plano com o cone e com a pirâmide.

Os OAs estão disponíveis no formato de vídeos. Os vídeos possuem áudio, explicando os conceitos que estão sendo 
estudados e podem ser baixados ${ }^{3}$ para serem utilizados sem acesso à internet. Também foram disponibilizados, junto aos vídeos, os arquivos das atividades feitas no GeoGebra 3D.

\section{4 - A sequência didática}

O material didático desenvolvido no formato de OAs para o estudo de volume de sólidos geométricos foi utilizado em uma sequência didática para turmas do ensino médio de duas escolas públicas, parceiras do Programa Institucional de Bolsa de Iniciação à Docência (PIBID).

A sequência didática foi planejada para considerar os conhecimentos prévios dos estudantes e propiciar a construção do conceito de volume e desenvolvimento de estratégias para o cálculo, em oposição ao modelo de ensino baseado na recepçãorepetição de informações.

A aplicação envolveu duzentos e trinta e cinco estudantes em sala de aula com a colaboração de professores em exercício, com duração total de doze horas-aula. As atividades foram desenvolvidas em momentos diferentes em cada escola. Na primeira escola as atividades foram desenvolvidas com vinte e cinco alunos de uma turma de segundo ano do ensino médio noturno, selecionada especialmente para a pesquisa. Enquanto que as atividades desenvolvidas na segunda escola, foram realizadas com duzentos e dez estudantes de todos os terceiros anos do ensino médio, como atividade do PIBID, inseridas em um dos temas do projeto didático "Matemática e Aplicações".

Para a análise dos conceitos prévios dos estudantes, em todas as turmas, foi aplicado um questionário investigativo e uma atividade de reconhecimento e classificação de embalagens.

O objetivo do questionário investigativo foi identificar se os estudantes eram capazes de reconhecer polígonos, resolver problemas com área de figuras planas e calcular área de superfície e volume de sólidos.

Além dos OAs foi elaborado e disponibilizado para os estudantes caderno de acompanhamento das atividades para que estes registrassem suas descobertas e conclusões, contendo situações-problema para resolução.

$\mathrm{Na}$ primeira escola, o caderno de acompanhamento foi recolhido ao fim de cada aula para análise dos dados. Já na segunda escola a avaliação ocorreu pela observação sistemática da utilização dos OAs e pela análise das respostas dos estudantes às questões relacionadas ao tema Geometria Espacial na atividade de competição, realizada como fechamento do projeto didático.

\footnotetext{
${ }^{3}$ www.unifal-mg.edu.br/matematica/?q=oa-ge
} 


\section{5 - Resultados e discussão}

A análise da atividade investigativa aplicada à turma de segundo ano da primeira escola revelou que a maioria dos estudantes não conseguia identificar polígonos e que a turma não tinha nenhum conhecimento sobre volume de sólidos. Por outro lado, os estudantes dos terceiros anos da segunda escola têm um conceito bastante simplista de área de figuras planas. Quando questionados sobre o significado de área, os estudantes relatam que área é "base vezes altura" ou "lado vezes lado", sem estabelecer qualquer relação com espaço ocupado e unidade de medida. Este fato evidencia a forma mecanicista como o assunto é tratado em muitas de nossas escolas.

Ao deduzir o volume do cubo e do bloco retangular, pôde-se notar a expressão de surpresa dos alunos ao entenderem que a obtenção do volume desses sólidos é realizada a partir do preenchimento completo destes sólidos, com as chamadas unidades de medida.

O uso dos OA foi fundamental na dedução das fórmulas de volumes. Mais do que chamar a atenção dos alunos para o estudo de volumes, esses objetos contribuíram para a visualização dos objetos geométricos, permitindo o entendimento dos conceitos. A possibilidade de movimentar o plano paralelo seccionando e comparando os diferentes tipos de sólidos geométricos de forma rápida e precisa, permitiu que os estudantes concluíssem por si mesmos que, a partir do Princípio de Cavalieri, é possível deduzir as fórmulas de volumes do paralelepípedo, cilindro, cone e esfera por comparação com o volume já deduzido do bloco retangular.

Apesar da destacada importância do Princípio de Cavalieri para o estudo de volumes de sólidos geométricos no ensino médio, é um resultado do qual muitos alunos nunca ouviram falar.

O OA para o estudo do volume da pirâmide possibilitou que os alunos visualizassem a decomposição do prisma. A manipulação das pirâmides uma a uma, foi essencial para o entendimento que a fórmula de volume da pirâmide ser um terço da área da base vezes a altura, sendo deduzida a partir do volume do prisma triangular, uma vez que essa representação nos livros didáticos não propicia uma visualização clara do que está sendo feito.

$\mathrm{Na}$ avaliação proposta em uma das escolas foi perguntado aos alunos a respeito da importância dos OA, durante o estudo de volumes de sólidos geométricos. Dos dezessete alunos doze responderam que foi importante o uso dessas animações para o entendimento dos conceitos.

Ao final da intervenção, os estudantes foram capazes de identificar e classificar embalagens com formatos geométricos diversos, como poliedros e corpos redondos, e calcular seus volumes. 
A sequência didática, com a utilização de recursos computacionais adequados, permitiu aos alunos o desenvolvimento de habilidades para identificar características de figuras planas e espaciais, resolver situaçõesproblema que envolvam conhecimentos geométricos e identificar relações entre grandezas e unidades de medida.

\section{6 - Considerações Finais}

Os objetos de aprendizagem desenvolvidos e aplicados na pesquisa juntamente com uma sequência didática para o estudo de volumes foi importante para a ideia inicial de volume, feita a partir da comparação com a unidade de medida, e a dedução das fórmulas de volume de diferentes sólidos geométricos, pelo Princípio de Cavalieri, conceitos que em geral, não são evidenciados no estudo de volumes.

A possibilidade de manipulação desses objetos contribui para que os estudantes possam construir, visualizar e formalizar os conceitos estudados. A manipulação confere a eles a autonomia na construção do próprio conhecimento.

Assim, a intervenção em situação real de sala de aula no ensino médio contribuiu para a aprendizagem real e significativa dos alunos, com o desenvolvimento de algumas habilidades da área de Matemática indicadas pelos Parâmetros Curriculares Nacionais para o Ensino Médio (BRASIL, 2002), relacionadas à competência em investigação e compreensão, como:

- formular hipóteses e prever resultados;

- selecionar estratégias de resolução de problemas;

- interpretar e criticar resultados numa situação concreta;

- distinguir e utilizar raciocínios dedutivos e indutivos;

- fazer e validar conjecturas, experimentando, recorrendo a modelos e relações;

- discutir ideias e produzir argumentos.

Este trabalho mostrou que é possível propor e aplicar uma sequência didática para o ensino médio, tendo como foco o trabalho com a ideia intuitiva de volume, tendo por metodologia a investigação matemática.

Tais investigações seriam inviáveis apenas com lápis-e-papel, com esta tecnologia o professor gere e controla as conclusões. Assim, uma das contribuições que os recursos computacionais podem trazer para a aprendizagem e produção matemática, é ser instrumento de mediação das ações humanas, colocando o aprendiz como sujeito de sua própria aprendizagem. E, principalmente, fomentando modos mais envolventes $\mathrm{e}$ críticos de aprender. 


\section{7 - Agradecimentos}

O presente trabalho foi realizado com apoio financeiro da Fundação de Amparo a Pesquisa do Estado de Minas Gerais (FAPEMIG), através do projeto de pesquisa processo SHA - APQ-04329-10 e do PIBID/CAPES.

\section{8 - Referências}

ALVES, G. S.; SOARES, A. B.; LIMA, C. Um estudo sobre o desenvolvimento do raciocínio espacial no ensino médio através da utilização do software Calques 3D. In: CONGRESSO BRASILEIRO DE INFORMÁTICA NA EDUCAÇÃO, XXV, 2005. São Leopoldo. Anais... Sociedade Brasileira de Computação, p. 2815-2823.

ALVES, G. Um estudo sobre o desenvolvimento da visualização geométrica com o uso do computador. In: SIMPÓSIO BRASILEIRO DE INFORMÁTICA NA EDUCAÇÃO, XVIII, 2007. São Paulo. Anais... Sociedade Brasileira de Computação, p. 1-10.

AUSUBEL, D. P.; NOVAK, J. D.; HANESIAN, H. Tradução: NICK, E. Psicologia educacional. Rio de Janeiro: Interamericana, 1980.

BRASIL. Ensino Médio orientações educacionais complementares aos Parâmetros Curriculares Nacionais. Brasília: MEC, 2002.

CARDOSO, A.; SOUZA JR, J. C. Conteúdos didáticos digitais para aprendizagem de funções. In: CONGRESSO BRASILEIRO DE INFORMÁTICA NA EDUCAÇÃO, XXII, 2011. Aracaju. Anais... Sociedade Brasileira de Computação, p. 371-379.
ENGERS, E. M. B. et al. Aplicações de uma Proposta para a Utilização do Aplicativo LOGO 3D no Processo de EnsinoAprendizagem da Geometria. In: CONGRESSO BRASILEIRO DE INFORMÁTICA NA EDUCAÇÃO, XV, 2004. Manaus. Anais... Sociedade Brasileira de Computação, p. 544-552.

JAHN, A. P.; BONGIOVANNI, V. Computador na sala de aula: Algumas possibilidades do software Cabri 3D para o estudo da Geometria Espacial. Revista do Professor de Matemática, Rio de Janeiro, n. 69, p. 50-54, 2009.

LIMA, E. L. Medida e Forma em Geometria. Coleção do Professor de Matemática. Rio de Janeiro: SBM, 2009.

PATERLINI, R. R. Os 'teoremas' de Cavalieri. Revista do Professor de Matemática, Rio de Janeiro, n. 72, p. 43-47, 2010.

TAROUCO, L. M. R. et al. Vídeo Mashup para Sites de Bibliotecas Universitárias Implicações do Open Content no Projeto e Desenvolvimento de Objetos de Aprendizagem Reutilizáveis. In: CONGRESSO BRASILEIRO DE INFORMÁTICA NA EDUCAÇÃO, XXII, 2011. Aracaju. Anais... Sociedade Brasileira de Computação, p. 184-193. 